$11,14,19$

\title{
Упругие свойства и ангармонизм твердых тел
}

\author{
(C) Д.С. Сандитов \\ Бурятский государственный университет им. Доржи Банзарова, \\ Улан-Удэ, Россия \\ Институт физического материаловедения СО РАН, \\ Улан-Удэ, Россия \\ E-mail: Sanditov@bsu.ru
}

Поступила в Редакцию 5 марта 2021 г.

В окончательной редакции 5 марта 2021 г.

Принята к публикации 11 октября 2021 г.

Квадраты скоростей продольной и поперечной акустических волн в отдельности практически не связаны с ангармонизмом, а их отношение $\left(v_{L}^{2} / v_{S}^{2}\right)$ оказывается линейной функцией параметра Грюнайзена $\gamma-$ меры ангармонизма. Полученная зависимость $\left(v_{L}^{2} / v_{S}^{2}\right)$ от $\gamma$ находится в удовлетворительном согласии с экспериментальными данными. Связь величины $\left(v_{L}^{2} / v_{S}^{2}\right)$ с ангармонизмом объясняется через ее зависимость от отношения тангенциальной и нормальной жесткостей межатомной связи $\lambda$, которое является однозначной функцией параметра Грюнайзена $\lambda(\gamma)$. Взаимосвязь коэффициента Пуассона $\mu$ и параметра Грюнайзена $\gamma$, установленная Беломестных и Теслевой, может быть обоснована в рамках теории Пинеды (Pineda). Обращено внимание на природу формулы Леонтьева, выведенной непосредственно из определения параметра Грюнайзена путем усреднения частоты нормальных мод колебаний решетки. Рассмотрена связь между соотношениями Грюнайзена, Леонтьева и Беломестных-Теслевой. Обсуждается возможность корреляции между гармоническими и ангармоническими характеристиками твердых тел.

Ключевые слова: упругие свойства, параметр Грюнайзена, формулы Беломестных-Теслевой, Леонтьева, уравнение Грюнайзена, тангенциальная и нормальная жесткости межатомной связи, кристаллы, стекла.

DOI: 10.21883/FTT.2022.02.51936.045

\section{1. Введение}

Известно, что в твердых телах при малых смещениях атома из равновесного положения $x$ он совершает гармонические колебания с параболическим потенциалом $U(x)$, которому соответствует линейная зависимость силы межатомного взаимодействия $f(x)$ от смещения $x$. При значительных смещениях атома проявляется ангармонизм - отклонение силы $f(x)$ от линейной зависимости и атом совершает ангармонические колебания с кубическим потенциалом. Ангармонически колеблющиеся атомы и группы атомов вносят существенный вклад в тепловое расширение, теплопроводность и другие физические свойства твердых тел [1-6]. Нелинейность силы межатомного и межмолекулярного взаимодействия играет важную роль практически на всех стадиях деформирования и разрушения аморфных полимеров и других материалов. Рождение очага разрушения в твердых телах обусловлено главным образом ангармонизмом $[3,4]$.

Мерой ангармонизма служит параметр Грюнайзена, который определяется изменением частоты нормальных мод колебаний решетки $v_{i}$ в зависимости от изменения объема тела

$$
\gamma_{i}=-\frac{V}{v}\left(\frac{d v_{i}}{d V}\right)=-\frac{d \ln v_{i}}{d \ln V} .
$$

В большинстве случаев используется приближение, когда все нормальные моды колебаний имеют одинаковую зависимость частоты от объема: $\gamma=-d \ln v / d \ln V$.
Основным соотношением для экспериментального определения $\gamma$ является уравнение (закон, формула) Грюнайзена [1]:

$$
\gamma=\frac{\beta V B}{C_{V}}
$$

где $\beta$ - коэффициент объемного теплового расширения, $V$ - молярный объем, $B$ - изотермический модуль объемного сжатия, $C_{V}$ - молярная теплоемкость при постоянном объеме. Это соотношение выводится из уравнения состояния твердого тела, куда входит параметр Грюнайзена $\gamma$ как характеристика нелинейности силы межатомного взаимодействия и ангармонизма колебаний решетки.

Принято считать, что параметры теории упругости (модули упругости, коэффициент Пуассона) как гармонические линейные величины, как правило, не должны быть связаны с ангармонизмом. В частности, гармонический и ангармонический коэффициенты разложения потенциальной энергии решетки в ряд Тейлора обычно рассматриваются как независимые параметры. Тем не менее, время от времени появляются работы, указывающие на наличие вполне определенной связи между гармоническими и ангармоническими характеристиками твердых тел [7-15].

В последнее время наблюдается заметный интерес к связи упругих свойств с параметром Грюнайзена [7-13]. Так, например, Беломестных и Теслевой [7] сравнительно недавно установлено, что коэффициент Пуассо- 
на $\mu$ является однозначной функцией параметра Грюнайзена $\gamma$

$$
\gamma=\frac{3}{2}\left(\frac{1+\mu}{2-3 \mu}\right),
$$

причем установлено достаточно строго с привлечением известных положений теории упругости, термодинамики и физической акустики. Примечательно то обстоятельство, что формула Беломестных-Теслевой (3) находится в удовлетворительном согласии с уравнением Грюнайзена (2) [7-10] (см., например, рис. 1). Лучшее согласие наблюдается для квазиизотропных щелочногалоидных кристаллов с центральными силами взаимодействия частиц. Основная причина небольших отклонений для некоторых тел [7-9], скорее всего, обусловлено разбросом значений $\gamma$, полученных разными исследователями. В качестве примера отметим, что по трем разным источникам параметр Грюнайзена (2) для алюминия составляет 2.11, 2.43 и 2.34 [8]. Не исключено, что здесь некоторую роль играет анизотропия ряда кристаллов.

Бодряковым с соавторами [11] предложено термодинамическое обобщение понятия о параметре Грюнайзена. Обобщенный вариант этой величины также связан с упругими свойствами, в частности, с коэффициентом Пуассона.

Леонтьев [12] с помощью теории упругости, молекулярной физики, термодинамики и физической акустики усреднил частоту нормальных мод колебаний решетки $v_{i}$ и непосредственно из определения параметра Грюнайзена (1) вывел следующую формулу для величины $\gamma$

$$
\gamma=\frac{3}{2}\left(\frac{B_{A}}{\rho v_{k}^{2}}\right),
$$

где $\rho-$ плотность, $B_{A}-$ адиабатический модуль объемного сжатия, $v_{k}^{2}-$ квадрат средней квадратичной скорости звука

$$
v_{k}^{2}=\frac{v_{L}^{2}+2 v_{S}^{2}}{3},
$$

$v_{L}$ и $v_{S}-$ скорости продольной и поперечной акустических волн, соответственно. Формула Леонтьева (4) удовлетворительно согласуется с уравнением Грюнайзена (2) [10].

Соотношения Беломестных-Теслевой (3) и Леонтьева (4) привлекательны тем, что позволяют рассчитывать $\gamma$ по более доступным экспериментальным данным, чем уравнение Грюнайзена (2). Вместе с тем обращает внимание то обстоятельство, что в их левых частях равенств находится мера ангармонизма $\gamma$, а в правые части входят на первый взгляд только гармонические (линейные) характеристики твердых тел $\left(\rho, B_{A}, v_{k}^{2}, \mu\right)$. Тем самым как бы обнаруживается противоречие.

Настоящая работа посвящена исследованию связи между упругими свойствами и параметром Грюнайзена в качестве примера связи между гармоническими и ангармоническими характеристиками твердых тел. Развито

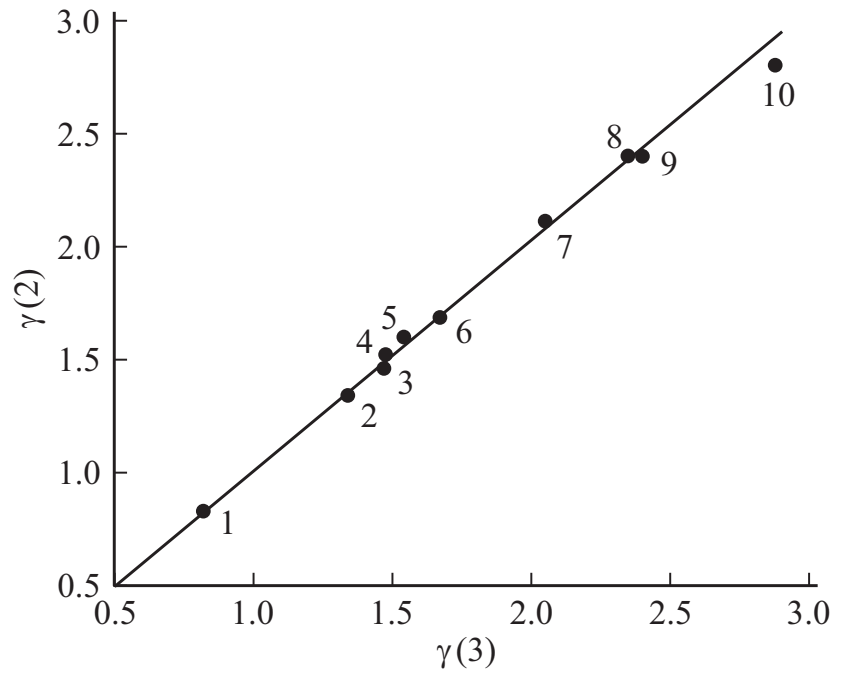

Рис. 1. Линейная корреляция между значениями $\gamma$, полученными по уравнению Грюнайзена $\gamma(2)$ и по формуле Беломестных-Теслевой $\gamma(3)$, для различных кристаллов (использованы данные [7-9]). $1-\mathrm{Be}, 2-\mathrm{LiF}, 3-\mathrm{NaCl}$, $4-\mathrm{LiCl}, 5-\mathrm{KCl}, 6-\mathrm{KBr}, 7-\mathrm{Al}, 8-\mathrm{Ag}, 9-\mathrm{Pb}$, $10-\mathrm{Au}$.

представление о том, что в формулах БеломестныхТеслевой и Леонтьева правые части равенств зависят от ангармонизма через зависимость отношения квадратов скоростей звука $\left(v_{L}^{2} / v_{S}^{2}\right)$ от параметра Грюнайзена. Наряду с оригинальными разработками кратко обсуждаются малоизвестные, но интересные работы по тематике исследования.

\section{2. Линейная зависимость $\left(v_{L}^{2} / v_{S}^{2}\right)$ от параметра Грюнайзена}

При изучении формул (3) и (4) обнаруживается тот факт, что их правые части являются функциями отношения квадратов скоростей распространения продольной и поперечной акустических волн $\left(v_{L}^{2} / v_{S}^{2}\right)$. Так, например, в уравнении Леонтьева (4) за счет величины $v_{\kappa}^{2}$ правая часть равенства оказывается функцией указанного отношения $\left(v_{L} / v_{S}\right)^{2}$ (см. соотношение (5))

$$
v_{\kappa}^{2}=\frac{v_{S}^{2}}{3}\left[\left(\frac{v_{L}}{v_{S}}\right)^{2}+2\right] .
$$

Далее, в правой части уравнения Беломестных-Теслевой (3) коэффициент Пуассона $\mu$, согласно известной формуле теории упругости [16], также является функцией отношения квадратов скоростей звука $\left(v_{L}^{2} / v_{S}^{2}\right)$

$$
\mu=\frac{2-\left(v_{L} / v_{S}\right)^{2}}{2-2\left(v_{L} / v_{S}\right)^{2}} .
$$


Таблица 1. Скорости звука, коэффициент Пуассона и параметр Грюнайзена неорганических веществ при стандартных условиях $\left(p=10^{5}\right.$ Па и $\left.T=298 \mathrm{~K}\right)$

\begin{tabular}{|c|c|c|c|c|c|c|c|c|}
\hline \multirow{2}{*}{ № } & \multirow{2}{*}{$\begin{array}{l}\text { Элементы и } \\
\text { соединения }\end{array}$} & \multicolumn{2}{|c|}{ Скорость звука, m/s } & \multirow{2}{*}{$\begin{array}{c}\text { Отношение квадратов } \\
\text { скоростей, }\left(v_{L} / v_{S}\right)^{2}\end{array}$} & \multirow{2}{*}{$\begin{array}{c}\text { Коэффициент } \\
\text { Пуассона, } \mu\end{array}$} & \multirow{2}{*}{$\begin{array}{c}\text { Параметр } \\
\text { Грюнайзена, } \gamma\end{array}$} & \multirow{2}{*}{$\frac{A}{(14)}$} & \multirow{2}{*}{$\frac{\bar{D}_{I}}{(16)}$} \\
\hline & & $v_{L}$ & $v_{S}$ & & & & & \\
\hline 1 & $\mathrm{LiF}$ & 7323 & 4518 & 2.627 & 0.200 & 1.34 & 0.63 & 1.5 \\
\hline 2 & $\mathrm{NaCl}$ & 4666 & 2755 & 2.869 & 0.243 & 1.46 & 0.59 & 1.7 \\
\hline 3 & $\mathrm{LiCl}$ & 5260 & 3058 & 2.959 & 0.245 & 1.52 & 0.61 & 1.6 \\
\hline 4 & $\mathrm{NaBr}$ & 3284 & 1885 & 3.35 & 0.270 & 1.56 & 0.55 & 1.7 \\
\hline 5 & $\mathrm{KCl}$ & 4090 & 2312 & 3.130 & 0.259 & 1.60 & 0.58 & 1.7 \\
\hline 6 & $\mathrm{KI}$ & 2623 & 1469 & 3.188 & 0.265 & 1.63 & 0.58 & 1.7 \\
\hline 7 & W & 5233 & 2860 & 3.348 & 0.283 & 1.62 & 0.56 & 1.8 \\
\hline 8 & $\mathrm{Fe}$ & 6064 & 3325 & 3.326 & 0.292 & 1.68 & 0.56 & 1.8 \\
\hline 9 & $\mathrm{KF}$ & 4641 & 2587 & 3.218 & 0.274 & 1.73 & 0.57 & 1.7 \\
\hline 10 & $\mathrm{RbI}$ & 2245 & 1198 & 3.512 & 0.309 & 1.73 & 0.53 & 1.9 \\
\hline 11 & $\mathrm{Co}$ & 5827 & 3049 & 3.652 & 0.357 & 1.87 & 0.53 & 1.9 \\
\hline 12 & $\mathrm{Cu}$ & 4726 & 2298 & 4.229 & 0.350 & 2.00 & 0.48 & 2.1 \\
\hline 13 & $\mathrm{Ag}$ & 3686 & 1677 & 4.831 & 0.379 & 2.40 & 0.45 & 2.2 \\
\hline 14 & $\mathrm{Pt}$ & 3960 & 1670 & 5.623 & 0.390 & 2.54 & 0.40 & 2.5 \\
\hline 15 & $\mathrm{~Pb}$ & 2158 & 860 & 6.30 & 0.372 & 2.93 & 0.43 & 2.3 \\
\hline
\end{tabular}

Таблица 2. Плотность $\rho$, скорости распространения продольных $\left(v_{L}\right)$ и поперечных $\left(v_{S}\right)$ акустических волн, модуль объемного сжатия $B_{A}$, коэффициента Пуассона $\mu$ и параметр Грюнайзена $\gamma$ для стекол $\mathrm{Na}_{2} \mathrm{O}-\mathrm{Al}_{2} \mathrm{O}_{3}-\mathrm{SiO}_{2}$. (Использованы данные [17])

\begin{tabular}{|c|c|c|c|c|c|c|c|c|c|c|c|}
\hline \multirow{2}{*}{ № } & \multicolumn{3}{|c|}{ Состав по синтезу, mol.\% } & \multirow{2}{*}{$\begin{array}{c}\rho, \\
\mathrm{kg} / \mathrm{m}^{3}\end{array}$} & \multirow{2}{*}{$\begin{array}{c}v_{L} \\
\mathrm{~m} / \mathrm{s}\end{array}$} & \multirow{2}{*}{$\begin{array}{c}v_{S} \\
\mathrm{~m} / \mathrm{s}\end{array}$} & \multirow{2}{*}{$\begin{array}{c}B_{A} \cdot 10^{-8}, \\
\mathrm{~Pa}\end{array}$} & \multirow{2}{*}{$\mu$} & \multirow{2}{*}{$\gamma$} & $A$ & \multirow{2}{*}{$\frac{\bar{D}}{(16)}$} \\
\hline & $\mathrm{Na}_{2} \mathrm{O}$ & $\mathrm{Al}_{2} \mathrm{O}_{3}$ & $\mathrm{SiO}_{2}$ & & & & & & & (14) & \\
\hline 1 & 15 & 0 & 85 & 2339 & 5430 & 3340 & 342 & 0.196 & 1.28 & 0.65 & 1.5 \\
\hline 2 & 15 & 5 & 80 & 2358 & 5570 & 3390 & 370 & 0.206 & 1.31 & 0.64 & 1.6 \\
\hline 3 & 15 & 10 & 75 & 2410 & 5697 & 3510 & 386 & 0.194 & 1.26 & 0.65 & 1.5 \\
\hline 4 & 15 & 15 & 70 & 2465 & 5737 & 3469 & 416 & 0.212 & 1.34 & 0.63 & 1.6 \\
\hline 5 & 15 & 20 & 65 & 2428 & 5850 & 3540 & 425 & 0.211 & 1.34 & 0.63 & 1.6 \\
\hline 6 & 15 & 25 & 60 & 2472 & 6000 & 3568 & 470 & 0.226 & 1.40 & 0.62 & 1.6 \\
\hline 7 & 25 & 0 & 75 & 2439 & 5280 & 3140 & 359 & 0.226 & 1.40 & 0.62 & 1.6 \\
\hline 8 & 25 & 5 & 70 & 2455 & 5480 & 3240 & 394 & 0.231 & 1.41 & 0.62 & 1.6 \\
\hline 9 & 25 & 10 & 65 & 2461 & 5610 & 3330 & 411 & 0.228 & 1.40 & 0.62 & 1.6 \\
\hline 10 & 25 & 20 & 55 & 2470 & 5680 & 3450 & 405 & 0.208 & 1.32 & 0.64 & 1.6 \\
\hline 11 & 25 & 25 & 50 & 2499 & 5790 & 3490 & 432 & 0.215 & 1.35 & 0.63 & 1.6 \\
\hline 12 & 25 & 30 & 45 & 2519 & 6026 & 3556 & 490 & 0.233 & 1.43 & 0.62 & 1.6 \\
\hline 13 & 35 & 0 & 65 & 2497 & 5340 & 3070 & 398 & 0.253 & 1.52 & 0.60 & 1.7 \\
\hline 14 & 30 & 5 & 65 & 2486 & 5500 & 3200 & 413 & 0.244 & 1.47 & 0.61 & 1.6 \\
\hline 15 & 20 & 15 & 65 & 2450 & 5670 & 3490 & 390 & 0.195 & 1.28 & 0.65 & 1.5 \\
\hline 16 & 17.5 & 17.5 & 65 & 2447 & 5746 & 3458 & 418 & 0.216 & 1.35 & 0.63 & 1.6 \\
\hline
\end{tabular}

Отмеченное наблюдение в отношении рассматриваемых формул наводит на мысль о том, что их правые части, возможно, зависят от ангармонизма за счет отношения квадратов скоростей продольной и поперечной акустических волн $\left(v_{L}^{2} / v_{S}^{2}\right)$. В самом деле, наши исследования ряда металлов, ионных и молекулярных кристаллов (табл. 1) показали [10]: если между параметром Грюнайзена $\gamma$ и квадратами скоростей $v_{L}^{2}$ и $v_{S}^{2}$ в отдельности фактически нет определенной взаимосвязи (рис. 2), то их отношение $\left(v_{L}^{2} / v_{S}^{2}\right)$ оказывается линейной функцией параметра Грюнайзена $\gamma-$ меры ангармонизма (рис. 3). У стеклообразных твердых тел [17] наблюдается аналогичная линейная корреляция между отношением квадратов скоростей звуковых волн и параметром Грюнайзена (рис. 4, табл. 2).

\section{3. Теоретический вариант зависимости $\left(v_{L}^{2} / v_{S}^{2}\right)$ от $\gamma$}

На рис. 3 и 4 приводится линейная корреляция между величинами $\left(v_{L}^{2} / v_{S}^{2}\right)$ и $\gamma$, полученная эмпирически на основе экспериментальных данных. Представляет интерес установление взаимосвязи этих величин с помощью существующих теоретических уравнений в данной области. 


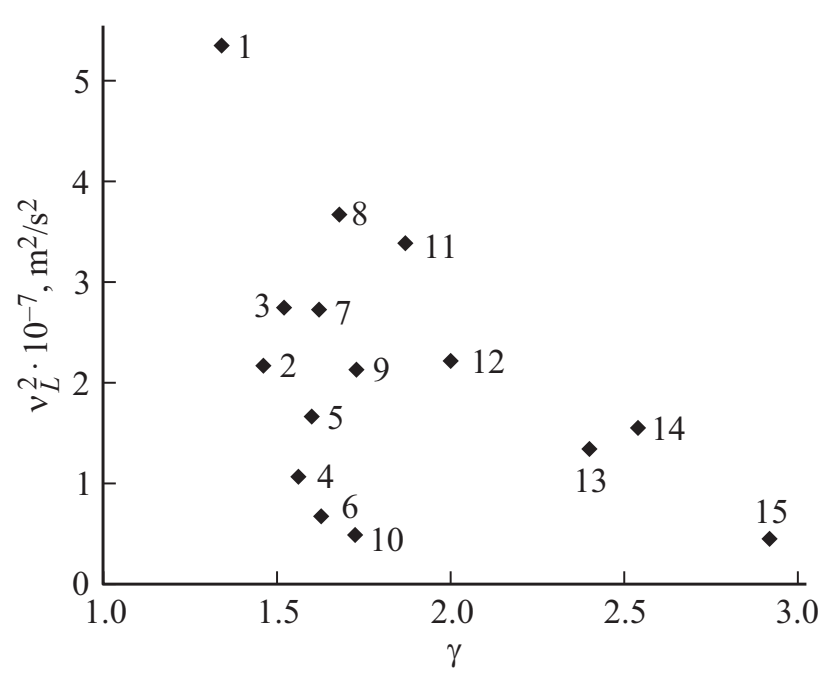

Рис. 2. Квадрат скорости продольной акустической волны $v_{L}^{2}$ в зависимости от параметра Грюнайзена $\gamma$. Номера точек соответствуют номерам кристаллов в табл. 1 .

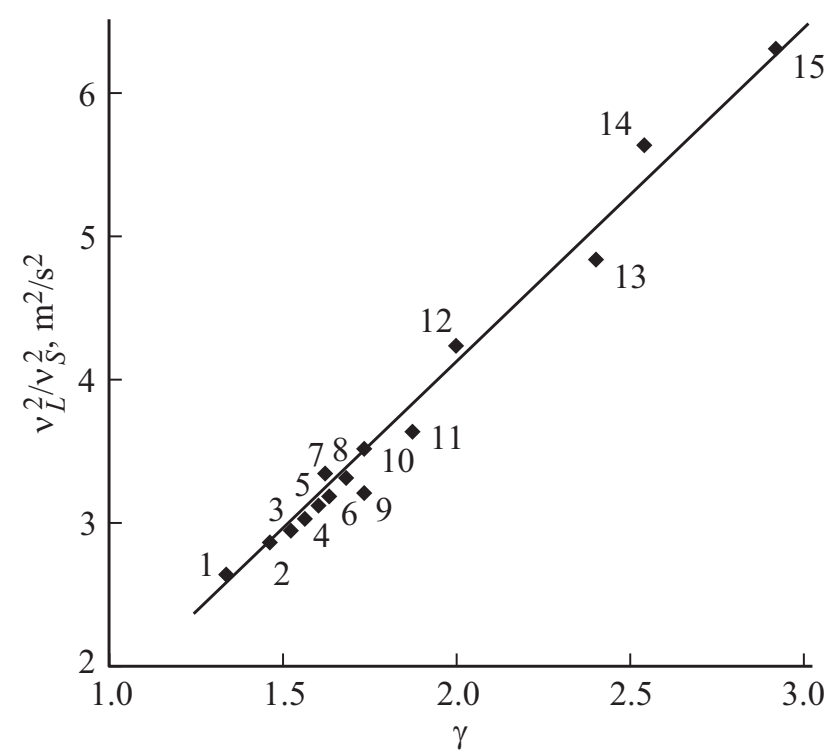

Рис. 3. Линейная корреляция между отношением квадратов продольной и поперечной скоростей звука $\left(v_{L}^{2} / v_{S}^{2}\right)$ и параметром Грюнайзена $\gamma$ для кристаллов, приведенных на рис. 2. Номера точек соответствуют номерам веществ в табл. 1.

Формулу для зависимости отношения $\left(v_{L}^{2} / v_{S}^{2}\right)$ от параметра Грюнайзена $\gamma$ можно вывести из уравнения Беломестных-Теслевой (3) и формулы теории упругости (6), которую разрешим относительно $\left(v_{L}^{2} / v_{S}^{2}\right)$ и запишем в виде [16]:

$$
\left(\frac{v_{L}}{v_{S}}\right)^{2}=\frac{2-2 \mu}{1-2 \mu}
$$

Выразив из уравнения Беломестных-Теслевой (3) коэффициент Пуассона $\mu$ через $\gamma$ и подставив его в формулу теории упругости (7), приходим к следующей зависимости отношения $\left(v_{L}^{2} / v_{S}^{2}\right)$ от $\gamma$

$$
\left(\frac{v_{L}}{v_{S}}\right)^{2}=4\left(\frac{3+\gamma}{9-2 \gamma}\right) \text {. }
$$

Такой же результат можно получить из формулы Беломестных для акустического параметра Грюнайзена [8].

Теоретическая зависимость (8) находится в согласии с экспериментальными данными для стекол - прямая на графике в координатах уравнения (8) проходит через начало координат с тангенсом угла наклона, равным единице (рис. 5). Исследованные кристаллы (табл. 1) в целом подчиняются зависимости (8), однако они по отношению к ней делятся на две группы, одна из которых

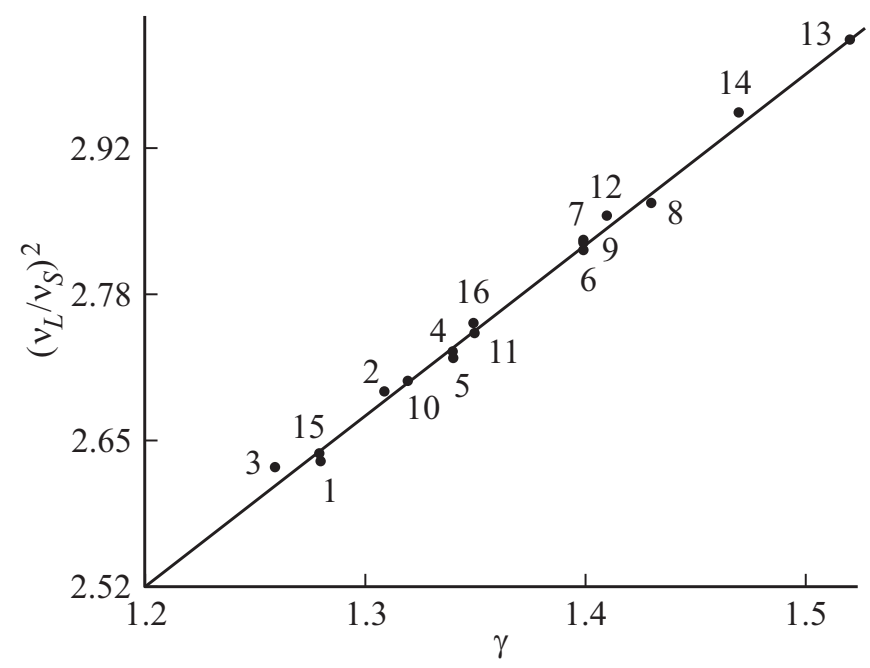

Рис. 4. Линейная корреляция между отношением квадратов продольной и поперечной скоростей звука $\left(v_{L}^{2} / v_{S}^{2}\right)$ и параметром Грюнайзена $\gamma$. Натриево-алюмосиликатные стекла с разным содержанием окислов. Номера точек соответствуют номерам стекол в табл. 2.

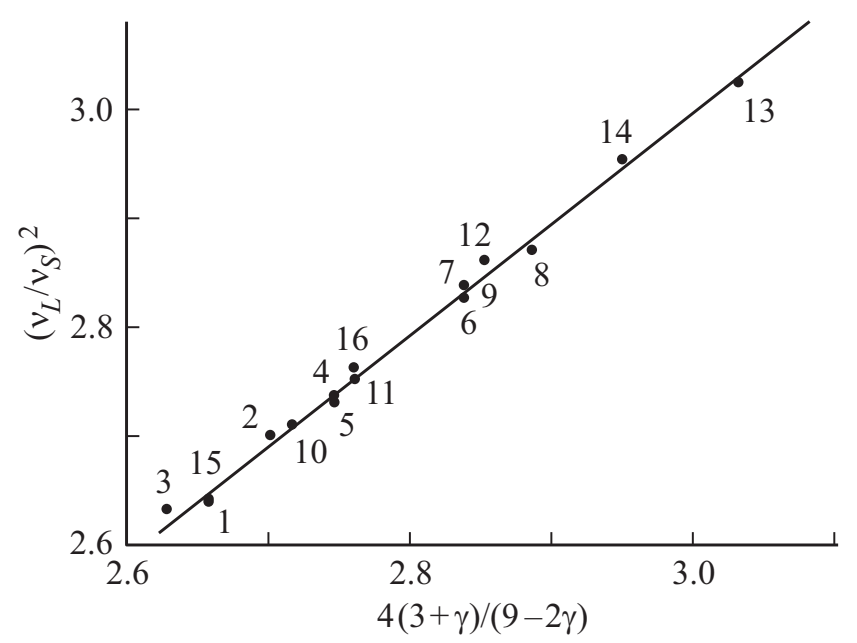

Рис. 5. Зависимость отношения квадратов скоростей звука $\left(v_{L}^{2} / v_{S}^{2}\right)$ от параметра Грюнайзена $\gamma$ в координатах, соответствующих уравнению (8). Натриево-алюмосиликатные стекла. Номера точек совпадают с номерами стекол в табл. 2. 

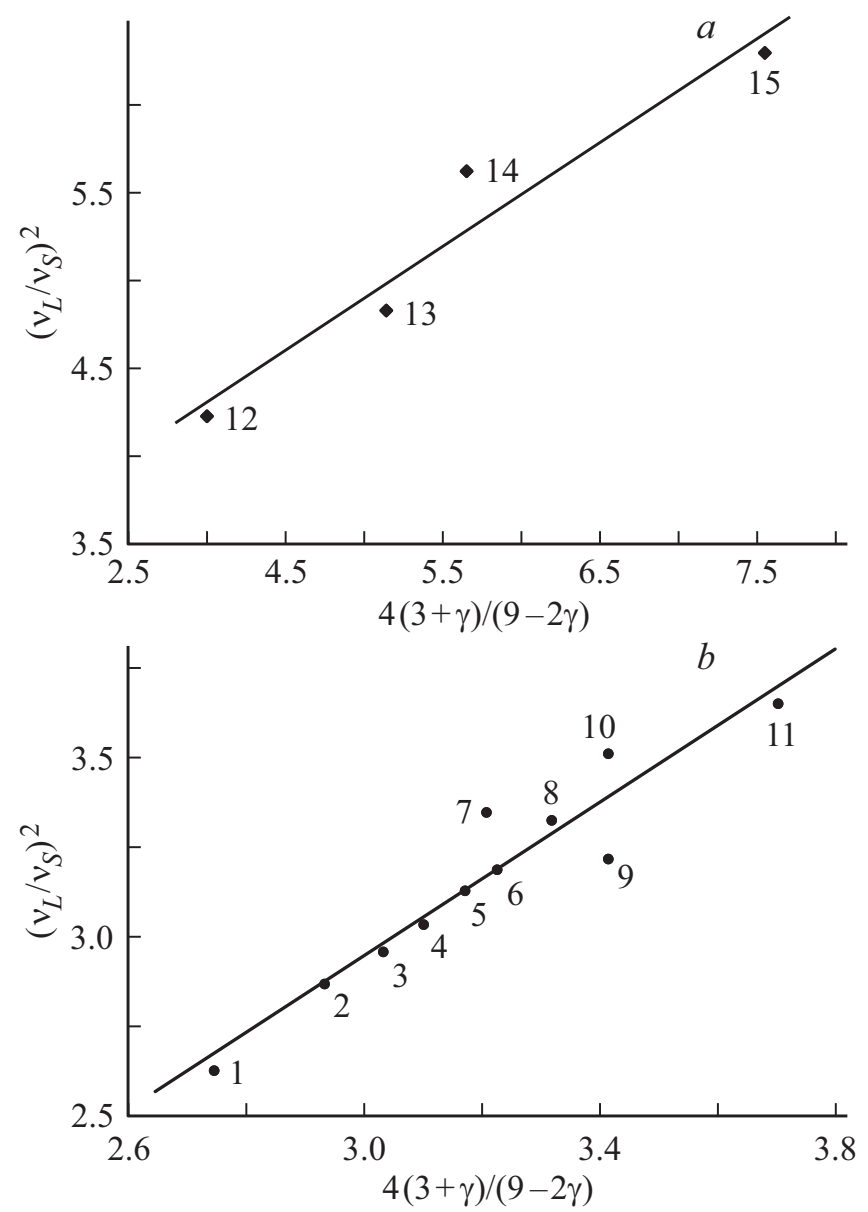

Рис. 6. Зависимость $\left(v_{L}^{2} / v_{S}^{2}\right)$ от параметра Грюнайзена $\gamma$ в координатах, соответствующих уравнению (8). Кристаллические твердые тела. Номера точек совпадают с номерами кристаллов в табл. 1.

описывается уравнением прямой, не проходящей через начало координат (рис. $6, a, b)$,

$$
\left(\frac{v_{L}}{v_{S}}\right)^{2}=a_{1}\left(\frac{3+\gamma}{9-2 \gamma}\right)+b_{1}
$$

где $a_{1}$ и $b_{1}$ - постоянные.

Возникает, естественно, вопрос, как согласовать соотношение $(8)$ с эмпирической линейной корреляцией, наблюдаемой между величинами $\left(v_{L}^{2} / v_{S}^{2}\right)$ и $\gamma$ (рис. 3 и 4). Оказывается, из формулы (8) можно получить линейную зависимость $\left(v_{L}^{2} / v_{S}^{2}\right)$ от $\gamma$ при условии $2 \gamma \ll 9$

$$
\left(\frac{v_{L}}{v_{S}}\right)^{2} \approx 1.3+0.4 \gamma
$$

Если для рассмотренных стекол, у которых $\gamma \approx 1.2-1.5$ (табл. 2), данное условие приемлемо, то для ряда исследованных кристаллов (табл. 1) оно выполняется с заметной натяжкой. Этот вопрос требует дальнейшего исследования с привлечением имеющихся разработок $[5-15,18]$.
Заметим, что, согласно формуле (8), соблюдается условие $(9-2 \gamma)>0$, откуда $\gamma<4.5$, что совпадает с максимальным параметром Грюнайзена при полиморфных превращениях в кристаллах. Например, при охлаждении кубической фазы кристалла цианида рубидия $\mathrm{RbCN}$ в температурном интервале от $T=380 \mathrm{~K}$ до окрестности температуры полиморфного превращения $T_{c}=110 \mathrm{~K}$ параметр Грюнайзена возрастает от $\gamma \approx 2$ до предельного значения $\gamma_{c}=4.5$ [19].

\section{4. О взаимосвязи уравнений Грюнайзена, Леонтьева и Беломестных-Теслевой}

Основная задача работы Леонтьева [12] заключалась в установлении связи между упругими и тепловыми свойствами веществ. Его формула (4) для параметра Грюнайзена предназначалась для использования при решении данной задачи.

Опираясь на основные положения термодинамики, теории упругости, молекулярной физики и физической акустики, Леонтьеву удалось установить связь тепловых характеристик $\beta$ и $C_{V}$ со скоростями распространения продольной и поперечной упругих волн (см. расшифровку $\left.v_{k}^{2}(5)\right)$

$$
\frac{C_{V}}{\beta V}=\frac{2}{3} \rho v_{k}^{2}
$$

Это соотношение находится в согласии с экспериментальными данными [10,12].

Мы обратили внимание на то обстоятельство, что из уравнения Грюнайзена (2) с привлечением равенства (10) выводится формула Леонтьева (4)

$$
\gamma=\frac{\beta V B}{C_{V}}=\left(\frac{\beta V}{C_{V}}\right) B=\frac{3}{2}\left(\frac{B}{\rho v_{k}^{2}}\right) \cong \frac{3}{2}\left(\frac{B_{A}}{\rho v_{k}^{2}}\right) .
$$

Здесь использовано известное приближение $B \approx B_{A}$. Отметим, что Леонтьев [12] выводит свою формулу (4) для $\gamma$ более строго без привлечения соотношения Грюнайзена (2).

Покажем, как из уравнения Грюнайзена (2) можно вывести формулу Беломестных-Теслевой (3). Умножив числитель и знаменатель данного уравнения (2) на модуль сдвига $G$ и принимая во внимание известную связь упругих модулей [16],:

$$
\frac{B}{G}=\frac{2}{3}\left(\frac{1+\mu}{1-2 \mu}\right),
$$

получаем следующую модификацию уравнения Грюнайзена (2)

$$
\gamma=A\left(\frac{1+\mu}{1-2 \mu}\right)
$$

где введено обозначение

$$
A=\frac{2}{3}\left(\frac{B V G}{C_{V}}\right)
$$


Используя соотношение Леонтьева (10) и модуль сдвига $G=\rho v_{S}^{2}$, множитель $A(12)$ представим в виде отношения квадратов скоростей звука

$$
A=\frac{v_{S}^{2}}{v_{k}^{2}} .
$$

Далее, из этого равенства (13) с помощью выражения (5) для $v_{k}^{2}$ и формулы (7) находим связь $A$ с коэффициентом Пуассона ти

$$
A=\frac{3}{2}\left(\frac{1-2 \mu}{2-3 \mu}\right) .
$$

Примечательно, что с учетом данного соотношения (14) модифицированное уравнение Грюнайзена (11) переходит в формулу Беломестных-Теслевой (3)

$$
\gamma=\left[\frac{3}{2}\left(\frac{1-2 \mu}{2-3 \mu}\right)\right]\left(\frac{1+\mu}{1-2 \mu}\right)=\frac{3}{2}\left(\frac{1+\mu}{2-3 \mu}\right) .
$$

Таким образом, с привлечением теории упругости из уравнения Грюнайзена (2) можно вывести формулу Беломестных-Теслевой (3), которая была получена авторами из иных исходных посылок [7].

Остановимся на физическом смысле множителя $A$ в уравнении (11).

Прежде всего обратим внимание на то обстоятельство, что коэффициент $A$ в выражении (13) показывает, какую долю составляет упругая энергия $\Delta U=\rho v_{S}^{2} V$, необходимая для деформации сдвига, от средней энергии межатомного взаимодействия $\bar{U}=\rho v_{k}^{2} V$

$$
A=\frac{v_{S}^{2}}{v_{k}^{2}}=\frac{\rho v_{S}^{2} V}{\rho v_{k}^{2} V}=\frac{G V}{\bar{U}}=\frac{\Delta U}{\bar{U}} .
$$

Поведение деформируемого тела определяется процессами образования и эволюции диссипативных структур, обеспечивающих рассеяние энергии, поступающей извне $[20,21]$. К особенностям таких структур относится существование универсальной иерархии пространственных масштабов (структурных уровней), что обусловлено фундаментальным свойством твердого тела - его сдвиговой устойчивостью, определяющей различие характерных пространственных масштабов областей локализации и рассеяния энергии, закачиваемой в деформируемое тело при внешнем воздействии.

Отношение пространственных масштабов диссипативных структур соседних уровней, иначе, размерность областей локализации энергии $D_{f}$, определяется соотношением (7) [20,21]:

$$
D_{f}=\frac{L_{i+1}}{L_{i}}=\frac{L_{l}}{L_{S}}=\frac{v_{L}^{2}}{v_{S}^{2}}=\frac{2(1-\mu)}{(1-2 \mu)},
$$

где $L_{i} \sim \rho \cdot v_{L}^{2}$ - характерный размер областей локализации энергии, $L_{S} \sim \rho \cdot v_{S}^{2}-$ характерный масштаб областей, в которых рассеивается энергия (за диссипацию энергии в деформируемом теле ответственны только сдвиговые напряжения [22]).

Если в этой формуле (15) величину $L_{l} \sim \rho \cdot v_{L}^{2}$ заменим усредненным характерным размером областей локализации энергии $\bar{L}_{l} \sim \rho \cdot v_{K}^{2}$, где $v_{K}-$ среднеквадратичная скорость звука (5), вместо $D_{f}=v_{L}^{2} / v_{S}^{2}$ получим некую среднюю размерность (см. (13) и (14))

$$
\bar{D}_{f}=\frac{v_{K}^{2}}{v_{S}^{2}}=\frac{2}{3}\left(\frac{2-3 \mu}{1-2 \mu}\right) .
$$

Как видно из сравнения выражений (13) и (16), множитель $A$ приобретает смысл обратной величины средней размерности областей локализации подводимой извне энергии

$$
A=\frac{v_{S}^{2}}{v_{K}^{2}}=\frac{1}{\bar{D}_{f}} .
$$

Если принять, что значение $\mu$ у разных твердых тел лежит примерно в интервале $0.165 \leq \mu \leq 0.475$ [20], то величина $\bar{D}_{f}$ может меняться в пределах $1.5 \leq \bar{D}_{f} \leq 7.7$, что вполне приемлемо, тогда как $D_{f}$ меняется в более широких пределах $2.5 \leq \bar{D} \leq 21$.

Таким образом, коэффициент $A$ в равенстве (11) можно рассматривать как обратную величину средней размерности областей локализации энергии, подводимой к деформируемым телам. Он равен доле энергии, которая рассеивается в процессе деформации.

Легко убедиться, что результаты расчетов $A$ по формулам (13) и (14) совпадают. В выражении (14) для множителя $A$ при изменении коэффициента Пуассона числитель и знаменатель меняются симбатно, поэтому величина $A$ слабо зависит от природы твердых тел и для группы тел одного структурного типа остается практически постоянной $A \approx$ const. Например у натриевоалюмосиликатных стекол $A$ составляет (табл. 2)

$$
A \approx \text { const } \approx 0.62-0.64,
$$

а средняя размерность областей локализации запасаемой энергии равна

$$
\bar{D}_{f} \approx \text { const } \approx 1.6-1.7
$$

Исследованные кристаллы (табл. 1) по значениям $\bar{D}_{f}=1 / A$ несколько условно можно разбить на две группы: 1) галоидные щелочные соли с $\mathrm{NaCl}$ - структурой $\bar{D}_{f} \approx 1.7(\mu=0.200-0.270)$ и 2$)$ металлы с гранецентрированной кубической структурой $\bar{D}_{f} \approx 2.1$ $(\mu=0.309-0.390)$.

Обращает внимание совпадение средних размерностей областей локализации запасаемой энергии $\bar{D}_{f}$ для ряда ионных кристаллов (табл. 1) и неорганических стекол (табл. 2), что согласуется с представлением Андерсона [23] об аналогии атомной динамики в стеклах и ионных солях.

Как и следовало ожидать в случае $A=$ const, в coответствии с уравнением (11) зависимость параметра Грюнайзена $\gamma$ от функции коэффициента Пуассона 


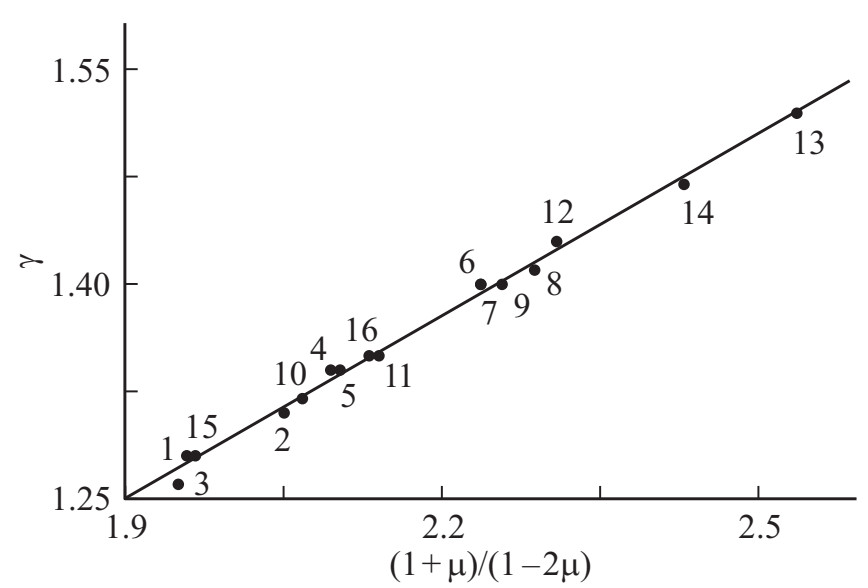

Рис. 7. Линейная зависимость параметра Грюнайзена $\gamma$ от функции коэффициента Пуассона $(1+\mu) /(1-2 \mu)$ для натриево-алюмосиликатных стекол. Номера точек совпадают с номерами стекол в табл. 2.

$(1+\mu) /(1-2 \mu)$ оказывается линейной (рис. 7). Этот факт подтверждает приближенное постоянство множителя $A$ в уравнении (11).

Используя формулу (5) для квадрата среднеквадратичной скорости звука $v_{k}^{2}$ и приведенные выше соотношения теории упругости, из уравнения Леонтьева (4) можно вывести формулу Беломестных-Теслевой (3) [10].

\section{5. О ранних подходах к связи коэффициента Пуассона с параметром Грюнайзена}

Взаимосвязь коэффициента Пуассона и параметра Грюнайзена типа (11) была установлена впервые в 1978 г. с помощью формул внутреннего давления $p_{i}$, обусловленного силами межатомного взаимодействия [24]. Величина $p_{i}$ определяется производной от внутренней энергии по объему при $T=$ const и выражает упругую реакцию решетки на объемное сжатие или растяжение. Поэтому внутреннее давление тесно связано с модулями упругости и деформацией решетки [25].

Из приближения закона Гука и предельной упругой деформации межатомной связи следует, что $p_{i}$ выражается соотношением [25]

$$
p_{1} \cong\left(\frac{1}{6 \gamma}\right) E,
$$

где $E$ - модуль упругости при одноосной деформации. Исходя из потенциала $M u$ и связи коэффициента Пуассона с параметрами данного потенциала, выводится аналогичная формула [24,26]:

$$
p_{1} \cong \frac{1-2 \mu}{6(1+\mu)} E .
$$

Сравнение этих двух равенств приводит к приближенной связи между параметром Грюнайзена и коэффициентом
Пуассона $[24,26]$ :

$$
\gamma \approx \frac{1+\mu}{1-2 \mu} .
$$

У ионных кубических кристаллов, например $(\mu \sim 0.20-0.25)$, оценка по этой формуле $\gamma \approx 2$ в первом приближении находится в согласии с результатами расчета $\gamma$ по уравнению Грюнайзена.

Как видим, формула (20) представляет собой частный случай соотношения (11) при $A \approx 1$. Для стеклообразных твердых тел уравнение (11) было получено ранее со следующей интерпретацией множителя $A[5,6,27]$ :

$$
A \approx \frac{2}{9} \ln \left(\frac{1}{f_{g}}\right),
$$

где $f_{g}=\left(V_{f} / V\right)_{T=T_{g}}$ - объемная доля флуктуационного свободного объема, замороженная при температуре стеклования $T_{g}$. Величина $f_{g}$ слабо зависит от природы стекол. У стеклообразных систем одного класса является фактически универсальной постоянной $f_{g} \approx$ const $[26,28,29]$. Оценка $A$ по этой формуле по порядку величины согласуется с результатами расчета по соотношениям (13) и (14).

\section{6. Микроскопическая трактовка параметра Грюнайзена. Предельная деформация межатомной связи}

Рассмотрим линейную цепочку связанных атомов (осцилляторов). Пусть $l$,Период решетки“ - расстояние между соседними атомами. При $l=l_{0}$ атом находится в равновесном положении. Через $a_{0}$ и $b_{0}$ обозначим соответственно гармонический и ангармонический коэффициенты разложения в ряд парного потенциала $U(r)$. Здесь $r$ - межатомное расстояние в двухатомной (парной) модели. Коэффициенты $a_{0}$ и $b_{0}$ определяются второй и третьей производными $U(r)$ в точке равновесия $r=r_{0}$.

Качественное представление о потенциале, в котором движутся атомы линейной цепи, можно получить, складывая потенциальные кривые, характеризующие взаимодействие атома с соседями справа и слева: $U_{-}$и $U_{+}$. Хотя каждая из них имеет собственный минимум (при $\left.r=r_{0}\right)$, вблизи которого

$$
U=U_{0}+\frac{a_{0}}{2}\left(r-r_{0}\right)^{2}-\frac{b_{0}}{6}\left(r-r_{0}\right)^{3}+\ldots,
$$

в сумме они дают потенциал $\Phi=U_{-}+U_{+}$, симметричный по смещению от положения, равноотстоящего от обоих соседей $[25,26,30]$. Возвращающее усилие $F$, приложенное к данному атому, также слагается из сил, действующих на него слева и справа $F=f_{-}+f_{+}$. Каждая из них определяется одной и той же производной парного потенциала $f(r)=d U / d r$. Используя разложение (21), можно установить, что вблизи узла решетки 
функция $f(r)$ выражается соотношением [25]:

$$
\begin{aligned}
f(r) & \approx a_{0}\left(r-l_{0}\right)-\frac{b_{0}}{2}\left(r-l_{0}\right)^{2} \\
& \approx c\left(l-l_{0}\right)^{2}+a(r-l)-\frac{b}{2}(r-l)^{2},
\end{aligned}
$$

где $c=a_{0}-b_{0}\left(l-l_{0}\right) / 2, b=b_{0}$,

$$
a=a_{0}-b_{0}\left(l-l_{0}\right) .
$$

Вообще говоря, $\Delta l=\left(l-l_{0}\right) \neq 0$, поскольку решетка может оказаться как в сжатом, так и в растянутом состоянии. Обычно $\Delta l / l_{0}$ настолько мало, что несовпадение минимумов $U_{-}$и $U_{+}$не мешает иметь по сумме параболическую форму, присущую гармоническому потенциалу. Поэтому перемещения атомов в твердом теле носят характер гармонических колебаний вблизи положения равновесия. При этом, как показал Дебай (см. $[2,25])$, частоты колебаний меняются в интервале от 0 до максимальной частоты $v_{m}$, которая выражается формулой

$$
v_{m}=\frac{1}{\pi} \sqrt{\frac{a}{m}} .
$$

Здесь $m$ - масса атома, величина $a$ определяется соотношением (23).

В теории Дебая параметр Грюнайзена выражается через характеристическую температуру $\theta_{D}=h v_{m}(h-$ постоянная Планка)

$$
\gamma=-\frac{d \ln \theta_{D}}{d \ln V}=-\frac{d \ln \left(h v_{m}\right)}{d \ln V} .
$$

В данную формулу подставим максимальную частоту $v_{m}$ из соотношения (24)

$$
\gamma=-\frac{d \ln \theta_{D}}{d \ln V}=-\frac{1}{2} \frac{d \ln a}{d \ln V}=-\frac{V}{2 a} \frac{d a}{d V} .
$$

При изотропной деформации имеем $d V / V=3(d l / l)$. Проводя дифференцирование в (25) с учетом этого равенства и выражения (23) для $a$, приходим к следующей формуле для параметра Грюнайзена

$$
\gamma=-\frac{l}{6 a} \frac{d a}{d l}=\frac{l b_{0}}{6 a} .
$$

Если принять во внимание, что имеет место равенство $l / a=l_{0} / a_{0}$, окончательно получаем микроскопическую интерпретацию $\gamma$ в виде $[2,25,31]$ :

$$
\gamma=\frac{l_{0} b_{0}}{6 a_{0}} .
$$

Как следовало ожидать, параметр Грюнайзена $\gamma$ пропорционален ангармоническому коэффициенту $b_{0}$, при $b_{0}=0$ он также равен нулю $\gamma=0$.

Приведенные результаты остаются в силе при переходе к трехмерному твердому телу. Я.И. Френкель [30, с. 171] для коэффициента теплового расширения трехмерного твердого тела предложил формулу, куда входят уже известные нам параметры $\left(a_{0}, b_{0}\right.$ и $\left.l_{0}\right)$

$$
\beta=\frac{b_{0} k}{2 a_{0} l_{0}^{2} B} .
$$

Умножив правую часть этого равенства на отношение $\left(3 N_{A} l_{0} / 3 N_{A} l_{0}\right)$, его можно представить в виде [31]:

$$
\beta=\left(\frac{l_{0} b_{0}}{6 a_{0}}\right) \frac{C_{V}}{B V},
$$

где $C_{V}=3 N_{A} k=3 R-$ молярная теплоемкость, $V=N_{A} l_{0}^{3}-$ молярный объем, $N_{A}-$ число Авогадро, $k-$ постоянная Больцмана, $R-$ газовая постоянная, $B-$ изотермический модуль объемного сжатия.

Из сравнения данного соотношения с уравнением Грюнайзена (2)

$$
\beta=\gamma \frac{C_{V}}{B V},
$$

получаем приведенную выше микроскопическую интерпретацию параметра Грюнайзена (26) [31] $\gamma=l_{0} b_{0} / 6 a_{0}$, которая оказывается справедливой для трехмерного твердого тела.

Остановимся на оценке предельной деформации межатомной связи $\Delta l_{m}=\left(l_{m}-l_{0}\right)$ в твердых телах. При $l=l_{m}$ в точке перегиба кривой потенциала сила взаимодействия атомов $f=-d U / d r$ проходит через максимум

$$
\left.\frac{d f}{d r}\right|_{r=l_{m}}=0 .
$$

Используя зависимость $f(r)$ в виде разложения в ряд (22), нетрудно видеть, что максимальное относительное удлинение связи между соседними атомами выражается следующим равенством

$$
\frac{\Delta l_{m}}{l_{0}}=\frac{a_{0}}{l_{0} b_{0}},
$$

которое с учетом микроскопической трактовки параметpa Грюнайзена (26) принимает вид $[25,26,32,33]$ :

$$
\frac{\Delta l_{m}}{l_{0}}=\frac{1}{6 \gamma} .
$$

Предельная деформация межатомной связи $(1 / 6 \gamma)$ входит в формулу внутреннего давления (18).

С привлечением зависимости $\gamma(\mu)$ по БеломестныхТеслевой (3) предельная деформация (27) оказывается однозначной функцией коэффициента Пуассона $\mu$

$$
\frac{\Delta l_{m}}{l_{0}}=\frac{2-3 \mu}{9(1+\mu)} .
$$

Расчет по этой формуле, например, для неорганических стекол (табл. 3) [34,35]

$$
\frac{\Delta l_{m}}{l_{0}} \cong 0.09-0.13
$$

находится в согласии с другими способами оценки данной деформации $[4,6,25,26]$. 
Таблица 3. Характеристики неорганических стекол (использованы данные [34,35])

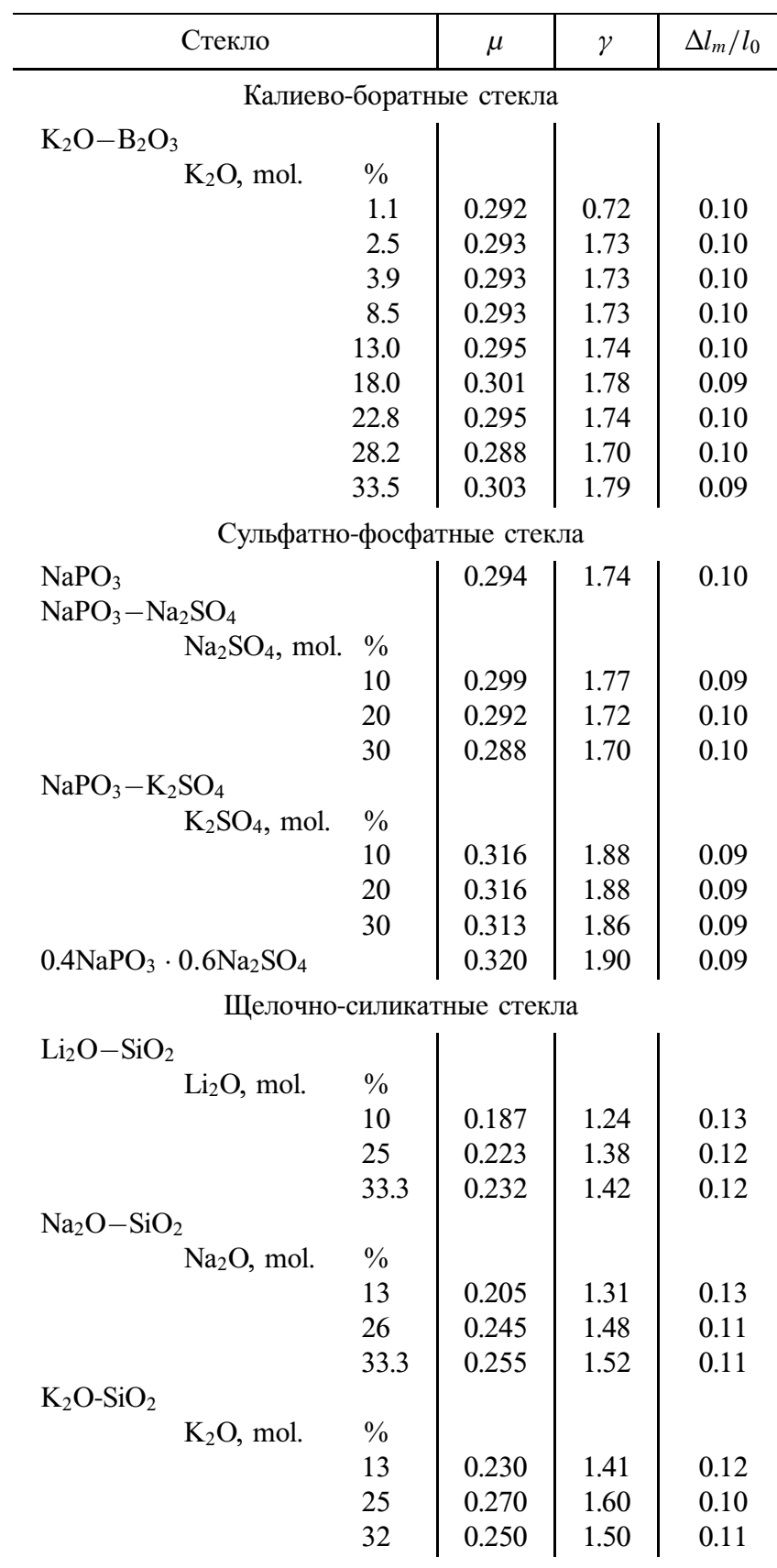

\section{7. Обсуждение результатов}

1. Полученные результаты рассмотрим с привлечением модели случайно упакованных атомов в виде сфер, взаимодействующих друг с другом в месте контакта двумя взаимно перпендикулярными силами: нормальными к плоскости контакта (центральными силами) $f_{n}=k_{n} x_{n}$ и тангенциальными (силами трения) $f_{t}=k_{t} x_{t}$. В рамках данной модели БРБ (Берлина-РотенбургаБасэрста) [36,37] коэффициент Пуассона определяется отношением тангенциальной $k_{t}$ и нормальной $k_{n}$ жест- костей межатомной связи $\lambda=\left(k_{t} / k_{n}\right)$ (формула БРБ)

$$
\mu=\frac{1-\lambda}{4+\lambda} .
$$

Из равенств (7) и (29) видно, что отношение квадратов звуковых скоростей $\left(v_{L}^{2} / \nu_{S}^{2}\right)$ определяется также микроскопическим параметром $\lambda$

$$
\left(\frac{v_{L}^{2}}{v_{S}^{2}}\right)=\frac{2(3+\lambda)}{2+3 \lambda}
$$

В свою очередь в соответствии с выражениями (3) и (29) параметр $\lambda$ связан с ангармонизмом $(\gamma)$

$$
\gamma=\frac{3}{2(1+\lambda)}
$$

С этих позиций связь величины $\left(v_{L}^{2} / v_{S}^{2}\right)$ с ангармонизмом объясняется ее зависимостью от относительной тангенциальной жесткости межатомной связи $\lambda=\left(k_{t} / k_{n}\right)$, которая является однозначной функцией параметра Грюнайзена $\gamma(31)$.

У аморфных органических полимеров и неорганических стекол очевидна зависимость параметра $\lambda$ от плотности поперечных межатомных связей, определяемой как число валентных связей на один катион $n$ (рис. 8) [38]. У линейных структур (поливинилхлорид, резина, селен), имеющих связанность 2 (два аниона, связанных с катионом вдоль цепи) имеем $n=0$ и $\mu \approx 0.4$, для линейно-разветвленных структур со связностью 3 $\left(\mathrm{B}_{2} \mathrm{O}_{3}, \mathrm{P}_{2} \mathrm{O}_{5}, \mathrm{As}_{2} \mathrm{O}_{3}\right) n=1$ и $\mu \approx 0.3$, для структурных сеток со связностью $4\left(\mathrm{SiO}_{2}, \mathrm{GeO}_{2}\right) n=2$ и $\mu \approx 0.15$.

Ясно, что с возрастанием плотности поперечных валентных связей $n$ (рис. 8) ослабляется нелинейность силы взаимодействия атомов, убывает ангармонизм $\gamma$ и в соответствии с зависимостью (31) растет относительная тангенциальная жесткость межатомной связи $\lambda$, что, согласно формуле БРБ (29), приводит к уменьшению коэффициента поперечной деформации $\mu$.

$\mathrm{B}$ аморфных полимерах на величины $\mu$ и $\gamma$ заметное влияние оказывают боковые разветвления основной цепи макромолекулы („боковые привески“ главной цепи). Минимальными значениями $\mu$ и $\gamma$ обладает полиэтилен, у которого роль боковой привески играет легкий атом водорода. Замещение его более крупным и тяжелым атомом фтора при переходе от полиэтилена к политетрафторэтилену приводит к возрастанию $\mu$ от 0.25 до 0.33 и, соответственно, к росту $\gamma$ от 3 до 4 [39].

При замещении легких атомов более крупными и тяжелыми атомами на боковых (а также концевых) участках цепи усиливается нелинейность силы межмолекулярного взаимодействия и ангармонизм колебаний решетки, растет параметр Грюнайзена $\gamma$. А это, согласно взаимосвязи $\lambda$ и $\gamma$ (31), снижает относительную тангенциальную жесткость связи $\lambda=\left(k_{t} / k_{n}\right)$, что приводит к росту коэффициента Пуассона $\mu$ (см. формулу БРБ (29)). 


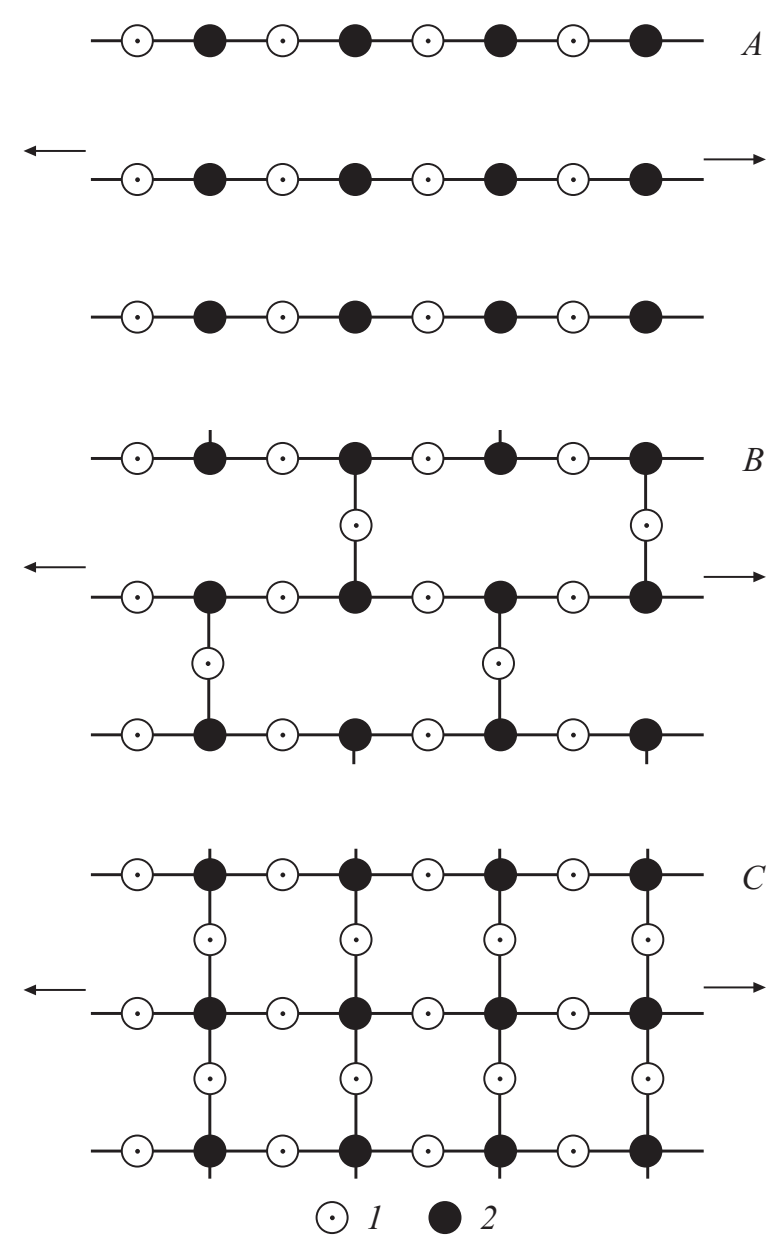

Рис. 8. Схема линейной $(A)$, линейно-разветвленной $(B)$ и сетчатой $(C)$ структур аморфных веществ [38]. 1 - анионы, 2 - катионы. $A-n=0, \mu \approx 0.4 ; B-n=1, \mu \approx 0.3$; $C-n=2, \mu \approx 0.15 . n-$ плотность поперечных связей. Стрелки - напряжения растяжения.

Таким образом, величины $\mu$ и $\gamma$ закономерно зависят от особенностей атомно-молекулярного строения стеклообразных твердых тел главным образом через микроскопический параметр $\lambda$.

Центральным силам $\left(k_{n} \gg k_{t}, \lambda \approx 0\right)$ соответствуют следующие значения коэффициента Пуассона и параметра Грюнайзена

$$
\mu=\frac{1-\lambda}{4+\lambda}=0.25 \text { и } \gamma=\frac{3}{2(1+\lambda)}=1.5 .
$$

В случае другого предельного значения $\lambda\left(k_{n} \ll k_{t}\right.$, $\lambda \approx \infty)$ имеем

$$
\mu=\frac{1-\lambda}{4+\lambda} \cong-1, \quad \gamma=\frac{3}{2(1+\lambda)} \approx 0 .
$$

Коэффициент Пуассона $\mu$ по определению равен отношению относительной поперечной деформации тела $(\Delta r / r)$ к его относительному продольному удлинению
$(\Delta l / l)$ при одноосном растяжении [16]:

$$
\mu=-\frac{\Delta r / r}{\Delta l / l} .
$$

При одноосном растяжении тела (стержня) происходит, как правило, его поперечное сжатие: $\Delta r<0$, поэтому $\mu>0$. В соответствии с определением $\mu$ (32) отрицательный коэффициент Пуассона $\mu<0$ означает поперечное расширение тела при его одноосном растяжении $\Delta r>0$, что, вообще говоря, противоречит здравому смыслу. Однако необходимо признать, что в последнее время появились публикации, подтверждающие существование изотропных тел с отрицательным коэффициентом поперечной деформации $\mu[36,37,40]$.

2. В формуле Леонтьева (4) произведение $\rho v_{k}^{2}$, обладающее характерными признаками упругих модулей, названо эффективным модулем упругости $[32,41]: K=\rho v_{k}^{2}$. Из соотношений теории упругости для кубических кристаллов (см., например, [12])

$$
B=\frac{C_{11}+2 C_{12}}{3} \quad \text { и } \quad \rho v_{k}^{2}=\frac{C_{11}+2 C_{44}}{3}
$$

видно, что при выполнении условия Коши $C_{12}=C_{44}$, когда между однородно деформированными областями кубической решетки действуют центральные силы, эффективный модуль упругости $K=\rho v_{k}^{2}$ совпадает с модулем объемного сжатия $K=B$. Во всех других случаях он отличен от $B$. Здесь $C_{11}, C_{12}$ и $C_{44}-$ упругие постоянные второго порядка.

В соответствии с формулой Леонтьева (4) параметр Грюнайзена определяется отношением модуля объемного сжатия и эффективного модуля упругости

$$
\gamma=\frac{3}{2}\left(\frac{B}{K}\right) \text {. }
$$

При выполнении условия Коши $K=B$ параметр Грюнайзена равен $\gamma=1.5$ и твердое тело находится в поле центральных сил, а при $K \neq B$ наблюдается отклонение от данного поля (от значения $\gamma=1.5$ ).

Из соотношений Леонтьева (4) и Беломестных-Теслевой (3) видно, что отношение $(B / K)$ оказывается однозначной функцией коэффициента Пуассона $\mu$, как и в случаях отношения других упругих модулей [16],

$$
\frac{B}{K}=\frac{1+\mu}{2-3 \mu} \text {. }
$$

Это выражение находится в согласии с экспериментальными данными как для кристаллов, так и для стеклообразных твердых тел (рис. 9 и 10 [42]). Можно также убедиться, что упругие модули в отдельности представляют собой гармонические характеристики твердых тел, а их отношения (например, $B / G$ ) оказываются однозначными функциями параметра Грюнайзена $\gamma-$ меры ангармонизма [см. зависимость $(B / G)$ от $\mu$ и в свою очередь $\mu$ от $\gamma$ ] (рис. 11,12$)$. По отношению к зависимости $(B / G)$ от $\gamma$ в отличие от изотропных 


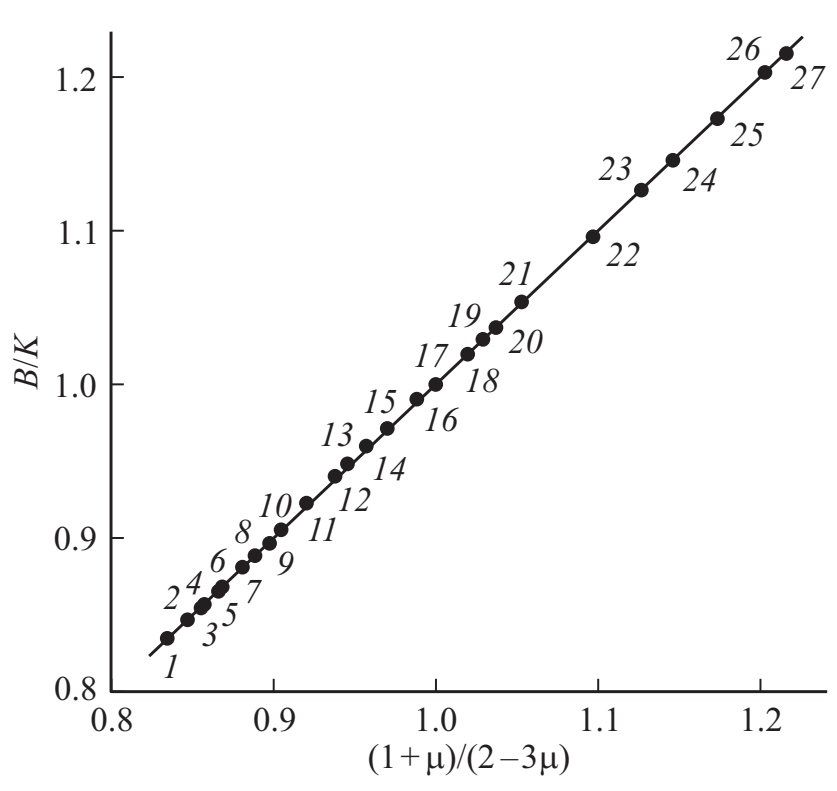

Рис. 9. Зависимость отношения модуля объемного сжатия к эффективному модулю упругости $(B / K)$ от функции коэффициента Пуассона $(1+\mu) /(2-3 \mu)$ для многокомпонентных оптических стекол: 1 - ЛК7, 2 - КФ6, 3 - Ф6, $4-$ КФ7, $5-\mathrm{K} 14,6-$ ЛФ5, $7-\mathrm{K} 8,8-\mathrm{K} Ф 4,9-\Phi 13,10-\mathrm{K} 19$, 11 - Ф4, 12 - ТФ1, 13 - БК6, 14 - БФ21, 15 - БФ8, 16 - БК 10,17 - ТФ7, 18 - ФК14, $19-$ ТК13, 20 - ТК23, 21 - БФ11, 22 - ТК17, 23 - ОФ2, 24 - СТК7, 25 - СТК9, 26 - ЛК4, 27 - ТБФ4. Данные взяты из справочника [42].

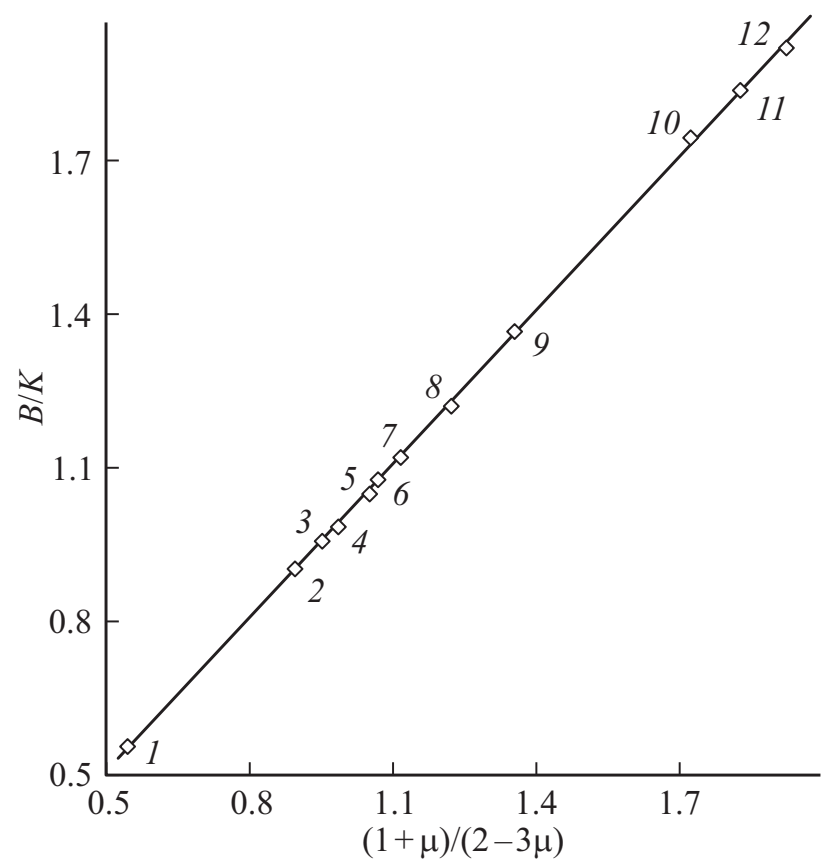

Рис. 10. Зависимость $(B / K)$ от функции коэффициента Пуассона $(1+\mu) /(2-3 \mu)$ для кристаллов. (Использованы данные [23]). $1-\mathrm{Be}, 2-\mathrm{LiF}, 3-\mathrm{NaF}, 4-\mathrm{LiCl}, 5-$ LiI, $6-\mathrm{NaClO}_{3}, 7-\mathrm{KBr}, 8-\mathrm{RbI}, 9-\mathrm{Ta}, 10-\mathrm{AgBr}$, $11-\mathrm{AgCl}, 12-\mathrm{Au}$.

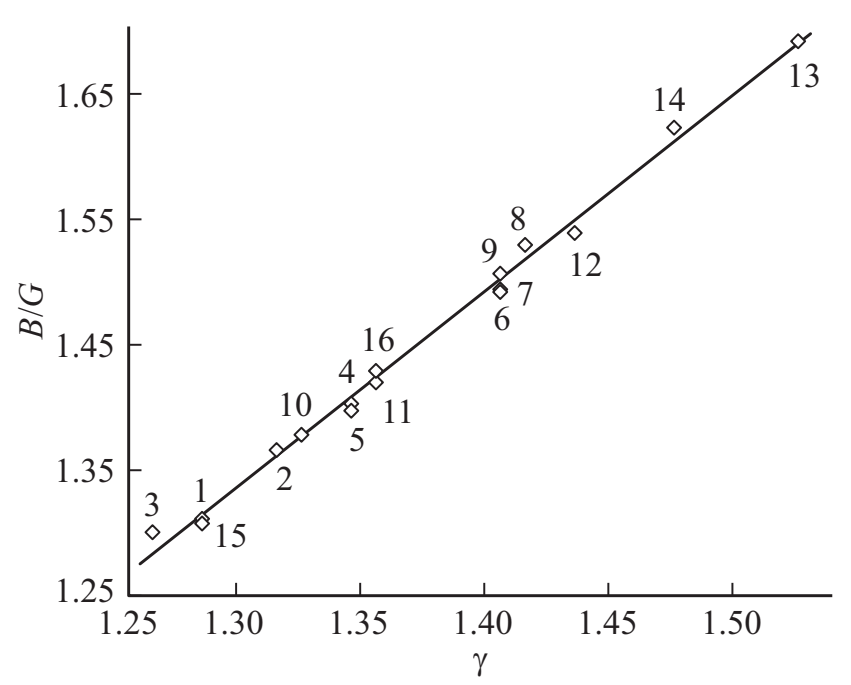

Рис. 11. Линейная корреляция между отношением модулей упругости $(B / G)$ и параметром Грюнайзена $\gamma$. Натриевоалюмосиликатные стекла $\mathrm{Na}_{2} \mathrm{O}-\mathrm{Al}_{2} \mathrm{O}_{3}-\mathrm{SiO}_{2}$ с разным содержанием окислов. Номера точек соответствуют номерам стекол в табл. $2 . B-$ модуль объемного сжатия, $G-$ модуль сдвига.

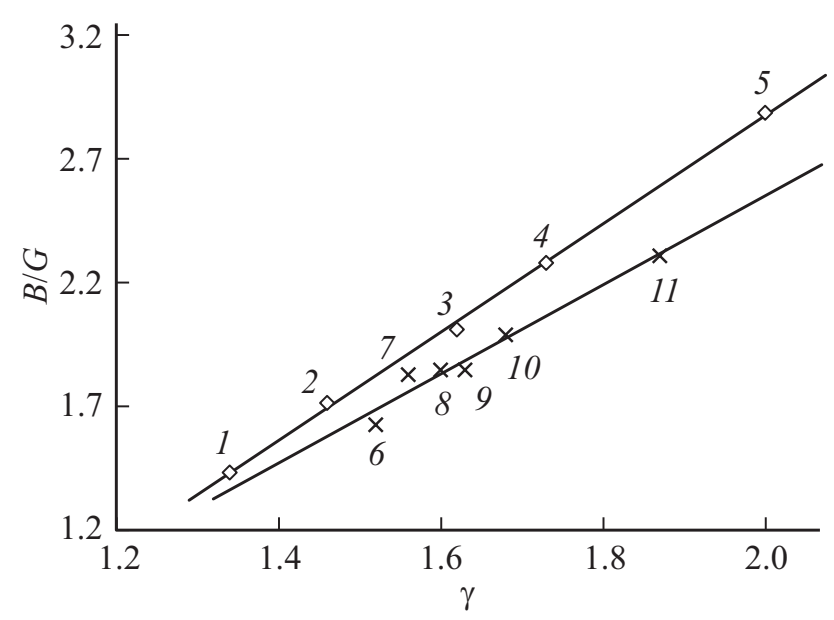

Рис. 12. Зависимость отношения модулей упругости $(B / G)$ от параметра Грюнайзена $\gamma$ для ряда кристаллов. $B-$ модуль объемного сжатия, $G-$ модуль сдвига. $1-\mathrm{LiF}, 2-\mathrm{NaCl}$, $3-\mathrm{W}, 4-\mathrm{RbI}, 5-\mathrm{Cu}, 6-\mathrm{LiCl}, 7-\mathrm{NaBr}, 8-$ $\mathrm{KCl}, 9-\mathrm{KI}, 10-\mathrm{Fe}, 11-\mathrm{Co}$. Использованы данные Беломестных-Теслевой [7].

стекол (рис. 11) рассмотренные кристаллы делятся на две группы (рис. 12).

3. Пинеда (Pineda) [13] теоретически исследовал влияние структурных изменений на упругие свойства металлических стекол. Он исходит из следующих трех основных предположений: (1) потенциал межатомного взаимодействия состоит из гармонической и ангармонической частей,

$$
U(r)=a\left(r-r_{0}\right)^{2}-b\left(r-r_{0}\right)^{3},
$$

где $a$ и $b-$ гармонический и ангармонический коэффициенты, соответственно, $r_{0}-$ межатомное 
расстояние, соответствующее минимуму потенциала, (2) распределение расстояний между ближайшими атомами является гауссовым, (3) упругие свойства определяются первой координационной сферой (непосредственным окружением атомов).

В окончательные довольно громоздкие формулы мгновенных модулей объемного сжатия $B$ и сдвига $G$ входят безразмерные параметры

$$
s=\frac{\delta}{r_{1}}, \quad \Delta=\frac{\delta_{1}}{r_{0}}, \quad \gamma_{1}=\frac{b r_{0}}{a},
$$

где $\delta=\left(r_{1}-r_{0}\right), r_{1}, \delta_{1}-$ средний радиус и ширина первой координационной сферы, соответственно. Величины $s$ и $\delta$ отражают отклонение межатомного расстояния от его равновесного значения $r_{0}$ и среднюю дисперсию вблизи $r_{0}$. Параметр $\gamma_{1}$ характеризует степень ангармоничности потенциала, он пропорционален параметру Грюнайзена $\gamma=b r_{0} / 6 a(26)$.

Теория была применена Пинедой для объяснения экспериментов по структурной релаксации и по всестороннему сжатию металлических стекол. В целом теория качественно правильно объясняет изменения упругих характеристик в этих опытах.

Теорию Пинеды мы использовали для проверки зависимости отношения упругих модулей $(B / G)$ и, следовательно, коэффициента Пуассона $\mu$ от параметра ангармоничности $\gamma_{1}$. Из теории следует, что такая зависимость существует. В самом деле, в соответствии с формулами упругие модули $B$ и $G$ пропорциональны гармоническому коэффициенту $a$ - параметру межатомного потенциала, а их отношение $(B / G)$ практически не зависит от $a$ и определяется главным образом параметром ангармоничности $\gamma_{1}$. Отсюда следует зависимость коэффициента Пуассона $\mu$ от параметра Грюнайзена $\mu-$ меры ангармонизма. Известно [16], что величина $\mu$ является однозначной функцией отношения упругих модулей $(B / G)$.

Таким образом, с точки зрения теории Пинеды получает определенное обоснование уравнение Беломестных-Теслевой (3), устанавливающее связь коэффициента Пуассона с параметром Грюнайзена.

4. Помимо однозначной связи коэффициента Пуассона с параметром Грюнайзена встречаются другие подобные примеры, например, эмпирическое правило Баркера [43], выражающее связь между модулем упругости $E$ и коэффициентом теплового расширения: $E \beta^{2}=$ const.

Представляет интерес подход Конторовой $[14,15]$ к интерпретации взаимосвязи линейных (гармонических) и нелинейных (ангармонических) величин. Как отмечалось выше, гармонический $a$ и ангармонический $b$ коэффициенты определены соответственно второй и третьей производными функции $U(r)$ при $r=r_{0}$ (см раздел 6). Используя в указанных производных величину $U(r)$ в виде потенциала $M u\left(U=-A r^{-m}+B r^{-n}\right)$ Конторова [14] установила взаимосвязь коэффициентов $a$ и $b$ в виде

$$
b=\frac{m+n+3}{2 r_{0}} a .
$$

Отсюда обсуждаемое явление объясняет существованием связи между $a$ и $b$ типа приведенного соотношения и полученной ею функциональной зависимости от них линейных и нелинейных свойств твердых тел.

Таким образом, работы Конторовой $[14,15]$ и Пинеды [13] указывают на принципиальную возможность реализации определенных корреляций между гармоническими и ангармоническими характеристиками твердых тел.

5. Согласно определениям $\mu$ и $\gamma$, коэффициент Пуассона $\mu$ и параметр Грюнайзена $\gamma$ характеризуют прежде всего изменение объема тела при деформации. Например, функция коэффициента Пуассона $(1-2 \mu)$ и, следовательно, сам коэффициент поперечной деформации $\mu$ связаны с изменением объема твердого тела при его одноосной деформации [44]:

$$
\frac{\Delta V}{V}=\varepsilon_{x}(1-2 \mu)
$$

где $\varepsilon_{x}$ - относительная одноосная деформация.

Кузьменко [18] считает, что коэффициент Пуассона служит характеристикой способности твердых тел противодействовать изменению их объема. Чем больше коэффициент поперечной деформации, тем меньше изменение объема твердого тела при деформации. Верхний предел $\mu=0.5$ следует из условия: изменение объема при деформировании полностью компенсируется противодействием вещества $(\Delta V=0)$. Это условие относится к жидкостям, а для твердых тел полной компенсации изменения объема не происходит, поэтому для них $\mu<0.5$.

В самом деле, как видно из равенства (37), чем больше коэффициент Пуассона данного тела, тем меньше его относительная объемная деформация $(\Delta V / V)$. При $\mu=0.5$ имеем $\Delta V / V=0$.

Применительно к параметру Грюнайзена можно использовать аналогичные рассуждения. Из определения $\gamma$ следует, что чем больше параметр Грюнайзена, тем меньше изменение объема тела, необходимое для изменения частоты колебаний решетки (при деформации),

$$
\frac{\Delta V}{V}=-\frac{1}{\gamma}\left(\frac{\Delta v}{v}\right) .
$$

C этой точки зрения параметр Грюнайзена так же, как и коэффициент Пуассона, характеризует способность твердого тела противодействовать изменению его объема. Из формулы Беломестных-Теслевой (3) следует, что максимальному значению коэффициента поперечной деформации $\mu=0.5$ соответствует предельное значение параметра Грюнайзена $\gamma_{\max }=4.5$. Как отмечалось в разделе 3, при структурных перестройках, в частности, при полиморфных превращениях в кристаллах, параметр Грюнайзена может достигать максимального значения 4.5. 
6. Обсуждение полученных результатов нами проведено на качественном уровне с помощью имеющихся в настоящее время теоретических разработок по тематике исследования. Обратим внимание на то обстоятельство, что по затронутым проблемам возникают вопросы, которые остаются без окончательного ответа. Во-первых, почему линейная (гармоническая) величина - параметр теории упругости $\mu$ оказывается однозначной функцией сугубо нелинейной (ангармонической) величины $\gamma$ ? Вовторых, почему у ряда изотропных твердых тел коэффициент Пуассона оказывается отрицательным? Это означает поперечное расширение тела при его одноосном растяжении, что противоречит здравому смыслу. В-третьих, почему формулы, относящиеся к анизотропным кубическим кристаллам, оказываются оправданными для изотропных стеклообразных твердых тел? Для полного ответа на подобные вопросы необходимо, чтобы появилась общепризнанная микроскопическая теория механических и тепловых свойств кристаллических и стеклообразных твердых тел.

\section{8. Заключение}

Развито представление о том, что в формулах Леонтьева (4) и Беломестных-Теслевой (3) правые части равенств зависят от ангармонизма через зависимость отношения квадратов продольной и поперечной скоростей звука от параметра Грюнайзена.

Получена формула для зависимости отношения квадратов скоростей звука $\left(v_{L}^{2} / v_{S}^{2}\right)$ от параметра Грюнайзена $\gamma$, которая находится в согласии с экспериментальными данными (по крайней мере для стеклообразных твердых тел). С привлечением модели БерлинаРотенбурга-Басэрста зависимость величины $\left(v_{L}^{2} / v_{S}^{2}\right)$ от ангармонизма $(\gamma)$ объясняется ее зависимостью от относительной тангенциальной жесткости межатомной связи $\lambda$, которая является однозначной функцией параметра Грюнайзена $\lambda(\gamma)$.

Показано, что из уравнения Грюнайзена можно вывести формулы Леонтьева и Беломестных-Теслевой. В модифицированном уравнении Грюнайзена (11) коэффициент $A$ характеризует, какую долю составляет упругая энергия, необходимая для деформации сдвига, от средней энергии межатомного взаимодействия. В качестве примера определенной корреляции между гармоническими и ангармоническими характеристиками твердых тел исследована связь между коэффициентом Пуассона $\mu$ - параметром теории упругости и параметром Грюнайзена $\gamma$ - мерой ангармонизма. Однозначная взаимосвязь коэффициента Пуассона и параметра Грюнайзена получает качественное обоснование в рамках теории Пинеды (Pineda).

С привлечением микроскопической трактовки параметра Грюнайзена установлено, что предельная упругая деформация межатомной связи является однозначной функцией коэффициента Пуассона.

\section{Благодарности}

Выражаю благодарность доцентам А.А. Машанову и М.Д. Дармаеву за помощь в подготовке работы к печати.

\section{Финансирование работы}

Работа выполнена при финансовой поддержке Министерства науки и высшего образования РФ (грант № 3.5406.2017/Б4).

\section{Конфликт интересов}

Автор заявляет, что у него нет конфликта интересов.

\section{Список литературы}

[1] Л. Жирифалько. Статистическая физика твердого тела. Мир, М. (1975). 382 с.

[2] Г. Лейбфрид. Микроскопическая теория механических и тепловых свойств кристаллов. Физматгиз, М.-Л. (1963). $312 \mathrm{c}$.

[3] С.Н. Журков, В.А. Петров. ДАН СССР 6, 1316 (1978).

[4] В.А. Петров, А.Я. Башкарев, В.И. Веттегрень. Физические основы прогнозирования долговечности конструкционных материалов. Политехника, СПб (1993). 475 с.

[5] Д.С. Сандитов, Г.В. Козлов. Физ. и хим. стекла 21, 549 (1995).

[6] Г.В. Козлов, Д.С. Сандитов. Ангармонические эффекты и физико-механические свойства полимеров. Наука, Новосибирск (1994). 260 с.

[7] В.Н. Беломестных, Е.П. Теслева. ЖТФ 74, 140 (2004).

[8] В.Н. Беломестных. Письма в ЖТФ 30, 14 (2004).

[9] Д.С. Сандитов, В.Н. Беломестных. ЖТФ 81, 77 (2011).

[10] Д.С. Сандитов, А.А. Машанов. ФТТ 63, 284 (2021).

[11] В.Ю. Бодряков, А.А. Повзнер, И.В. Сафронов. ЖТФ 76, 69 (2006).

[12] К.Л. Леонтьев. Акуст. журн. 27, 554 (1981).

[13] E. Pineda. Phys. Rev. B 73, 104109 (2006).

[14] Т.А. Конторова. В кн.: Некоторые проблемы прочности твердых тел. Изд-во АН СССР, М.-Л. (1959).

[15] В.П. Жузе, Т.А. Конторова. ЖТФ 28, 727 (1958).

[16] Л.Д. Ландау, Е.М. Лифшиц. Теория упругости. 3-е изд. Наука, М. (1965). 204 с.

[17] В.Я. Лившиц, Д.Г. Теннисон, С.Б. Гукасян, А.К. Костанян. Физ. и хим. стекла 8, 688 (1982).

[18] В.А. Кузьменко. Новые схемы деформирования твердых тел. Наук. думка, Киев (1973). 200 с.

[19] В.Н. Беломестных, Е.П. Теслева, Э.Г. Соболева. ЖТФ 79, 153 (2009).

[20] А.С. Баланкин. Письма в ЖТФ 16, 14 (1990).

[21] В.Б. Лазарев, А.С. Баланкин, А.Д. Изотов, А.А. Кажушко. Структурная устойчивость и динамическая прочность неорганических материалов. Наука, М. (1993). 175 с.

[22] Р.Г. Архипов. ЖЭТФ 92, 1021 (1987).

[23] O. Anderson. Physical Acoustics. V. III. Part B. Lattice Dynamics / Ed. W.P. Mason. Academic, N. Y.-London (1965).

[24] Д.С. Сандитов, Г.М. Бартенев, Ш.Б. Цыдыпов. Физ. и хим. стекла 4, 301 (1978).

[25] А.И. Бурштейн. Молекулярная физика. Наука, Новосибирск (1986). 288 с. 
[26] Д.С. Сандитов, Г.М. Бартенев. Физические свойства неупорядоченных структу. Наука, Новосибирск (1982). 259 с.

[27] Д.С. Сандитов, В.В. Мантатов. Физ. и хим. стекла 17, 174 (1991).

[28] Д.С. Сандитов. ЖЭТФ 142, 123 (2012).

[29] Д.С. Сандитов. ЖЭТФ 150, 501 (2016).

[30] Я.И. Френкель. Введение в теорию металлов. ОГИЗ, Л.-М. (1948). $291 \mathrm{c}$.

[31] Д.С. Сандитов, В.В. Мантатов. Физ. и хим. стекла 3, 287 (1983).

[32] Д.С. Сандитов. УФН 190, 355 (2020).

[33] С.Ш. Сангадиев, М.В. Дармаев, Д.С. Сандитов. Высокомолекуляр. соединения Сер. А 62, 170 (2020).

[34] О.В. Мазурин, М.В. Стрельцина, Т.Н. Швайко-Швайковская. Свойства стекол и стеклообразующих расплавов. Наука, Л.-СПб (1973-1998). Т. 1-6.

[35] MDL ( S SciGlass-7.8 Institute of Theoretical Chemistry, MA (2012).

[36] А.А. Берлин, Л. Ротенбург, Р. Басэрст. Высокомолекуляр. соединения. Сер. Б 33, 619 (1991).

[37] А.А. Берлин, Л. Ротенбург, Р. Басэрст. Высокомолекуляр. соединения. Сер. А 34, 6 (1992).

[38] B. Bridge, N.D. Patel, D.N. Waters. Phys. Status Solidi A 77, 655 (1983).

[39] И.И. Перепечко. Свойства полимеров при низких температурах. Химия, М. (1977). $271 \mathrm{c.}$

[40] Д.А. Конёк, К.В. Войцеховски, Ю.М. Плескачевский, С.В. Шилько. Механика композитных материалов и конструкций 10, 35 (2004).

[41] Д.С. Сандитов, М.В. Дармаев. Неорган. материалы 55, 660 (2019).

[42] Оптические стекла. Справочник/ Под ред. Г.Т. Петровского. Изд-во ГОИ им. С.И. Вавилова, Л. (1975).

[43] R. Barker. J. Appl. Phys. 34, 107 (1963).

[44] Г.П. Иванов, Т.А. Лебедев. Тр. Ленинградского политех. ин-та 236, 38 (1964).

Редактор Т.Н. Василевскал 